\title{
COVID-19 in a Child With Diabetic Ketoacidosis: An Instigator, a Deviator or a Spectator
}

$\mathrm{W}$ e report severe acute respiratory syndrome coronavirus 2 (SARS-CoV-2) precipitated diabetic ketoacidosis in a child with newly diagnosed type 1 diabetes mellitus with mild hyperinflammatory syndrome leading to fluid responsive shock.

A 15-year-old previously asymptomatic girl presented to the emergency department in the first week of May, 2020, with complaints of acute onset of abdominal pain and vomiting. At the referring hospital, she was noted to have hyperglycemia and severe metabolic acidosis ( $\mathrm{pH} 6.9$, bicarbonate $2 \mathrm{mEq} / \mathrm{L})$. She was initiated on a fluid bolus and was referred to our center.

On admission to the pediatric intensive care unit, the child was observed to be lethargic (GCS-14). On clinical examination, she had normal blood pressure with heart rate of 140/minute, cold extremities, tachypnea (respiratory rate 40/minute) and Kussmaul breathing. She had short stature (with a height of 145 $\mathrm{cm},<-2 \mathrm{SD})$ normal body mass index $\left(19 \mathrm{~kg} / \mathrm{m}^{2}\right)$, and nofeatures of insulin resistance. Systemic examination was unremarkable except for mild generalized abdominal tenderness. Her blood investigations revealed random blood sugar of $414 \mathrm{mg} / \mathrm{dL}$, neutrophilic leukocytosis, and serum potassium was $2 \mathrm{mEq} / \mathrm{L}$. Her urine showed 4+ ketones, and arterial blood gases were suggestive of severe compensated metabolic acidosis $(\mathrm{pH}=7.03)$. Her HbA1C was $13.5 \%$.

Fluid deficit replacement followed by insulin infusion at $0.1 \mathrm{unit} / \mathrm{kg} /$ hour was initiated. Over the next 6 hours, the blood sugars began normalizing at a rate of around $50 \mathrm{mg} / \mathrm{dL}$ per hour; however, severe metabolic acidosis persisted. This was accompanied with a clinical deterioration of sensorium and onset of shallow breathing pattern with a rapid rise in partial pressure of carbon dioxide $\left(\mathrm{pCO}_{2}\right)$ and oxygen desaturation on arterial blood gas, requiring the initiation of non-invasive ventilation. A chest radiograph at the time revealed low volume lung with mild bilateral haziness. In view of the possibility of cerebral edema, $3 \mathrm{~mL} / \mathrm{kg}$ of $3 \%$ sodium chloride was infused over 20 minutes, and fluid intake was optimized. With these measures, sensorium, $\mathrm{pCO}_{2}$ and oxygen saturation improved. The nasopharyngeal swab reverse transcriptase polymerase chain reaction, done as per institutional protocol for all inpatients, was positive for SARS-CoV-2. Oral hydroxychloroquine $(6.5 \mathrm{mg} / \mathrm{kg}$ twice daily for 1 day followed by 3.25 $\mathrm{mg} / \mathrm{kg}$ twice daily for 4 days) was added to the treatment regimen. She had a low grade fever on the second day of admission, which was managed symptomatically.
By the third day, the child's sensorium had significantly improved and glycemic control had been achieved and she was weaned to high flow nasal cannula. However, during the course of the day she developed tachycardia, decreased urine output and sudden onset hypotension requiring two normal saline boluses of $20 \mathrm{~mL}$ per $\mathrm{kg}$ to restore her circulatory status. Following the fluid resuscitation, there was worsening of the base line tachypnea without the requirement of supplemental oxygen. A rise in creatinine to a maximum of $2 \mathrm{mg} / \mathrm{dL}$ from a baseline of $0.4 \mathrm{mg} / \mathrm{dL}$ was also documented which took two days to normalize despite optimal fluid status maintained by intravenous fluids and nasogastric tube feeds. Despite good control of blood sugars and resolution of ketonuria, the child was noticed to have persistent severe metabolic acidosis and hyperchloremia which gradually improved over the next four days. By day 5, acidosis and appetite improved, hence she was switched over to 3-hourly subcutaneous insulin according to a sliding scale for the first day followed by basal bolus regimen and was discharged after 14 days of hospital stay.

There have been many reports on new onset diabetes in SARS-CoV-2 positive patients as well as worsening of glycemic control in those with preexisting diabetes mellitus [1]. However, majority of the world wide data point towards type 2 diabetes, with only a few anecdotal reports of COVID-19 infection in individuals with juvenile diabetes [2]. The expression of angiotensin converting enzyme 2 (ACE-2) receptors on pancreatic $\beta$ cells can lead to direct injury to pancreatic beta cells and decreased insulin secretion which might then precipitate ketoacidosis [3].Similar cases have been reported in the viremic phase of other viral illnesses like H1N1 too [4].

Multiple questions regarding the association of COVID-19 and diabetic ketoacidosis remain unanswered such as precipitation in a child with previously undiagnosed diabetes (suggested by a highly elevated HbAlc level); the cause of circulatory collapse despite adequate initial fluid resuscitation, and the mechanism of renal injury (prerenal) seen in the child. Although GAD antibodies were negative, absence of obesity, markers of insulin resistance and negative family history favored the clinical diagnosis of type 1 diabetes.

The COVID infection most probably also triggered the hyperinflammatory response in the child leading to third spacing and the fluid responsive shock with subsequent early acute tubular necrosis and mild acute kidney injury [5]. The circulatory collapse was observed to occur in the first 4-5 days of illness in the patient which probably coincides with the peak of viremia. The increased work of breathing during the fluid resuscitation also points towards the need of slower and judicious fluid resuscitation in diabetic ketoacidosis or shock, especially in the setting of COVID- related pulmonary capillary leak. Lastly, the hyperchloremic metabolic acidosis took more than 96 hours to get corrected in spite of tailoring the chloride content of iv fluid which is an unusual and atypical pattern. The 
above clinical presentation may fit into a pediatric inflammatory multisystem syndrome (PIMS) associated with COVID-19 [6]. However, further data is required to shed light on the complex and varying presentations of coronavirus infection in children with and without associated co-morbidities.

Published online: July 15, 2020; PII: S097475591600211.

Sanila Daniel, ${ }^{1 *}$ Bhushit Gadhiya, ${ }^{1}$ AKanksha PARIKH $^{2}$ AND Preetha Joshi ${ }^{1}$ Departments of ${ }^{1}$ Pediatric Intensive Care and ${ }^{2}$ Pediatrics, Kokilaben Dhirubhai Ambani Hospital and Research Institute, Mumbai, Maharashtra, India. *drsaniladaniel@gmail.com

\section{REFERENCES}

1. Chee YJ, Ng SJH, Yeoh E. Diabetic ketoacidosis precipitated by Covid-19 in a patient with newly diagnosed diabetes mellitus. Diabetes Res Clin Pract.
2020;164:108166.

2. Bornstein SR, Rubino F, Khunti K, Mingrone G, Hopkins $\mathrm{D}$, Birkenfeld AL, et al. Practical recommendations for the management of diabetes in patients with COVID-19. Lancet Diab Endocrinol. 2020;8:546-50.

3. Yang J, Lin S, Ji X, Guo LM. Binding of SARS coronavirus to its receptor damages islets and causes acute diabetes. Acta Diabetol. 2010;47:193-99.

4. Tan H, Wang C,Yu Y. H1N1 influenza: The trigger of diabetic ketoacidosis in a young woman with ketosis-prone diabetes. Am J Med Sci. 2012;343:180-83.

5. H Su, M Yang, C Wan, Yi LX, Tang F, Zhu HY, et al. Renal histopathological analysis of 26 postmortem findings of patients with COVID-19 in China. Kidney Int. 2020;98:219-27.

6. Royal College of Paediatrics and Child Health. Guidance: Paediatric multisystem inflammatory syndrome temporally associated with COVID-19. UK: Royal College of Paediatrics and Child Health; 2020. 